
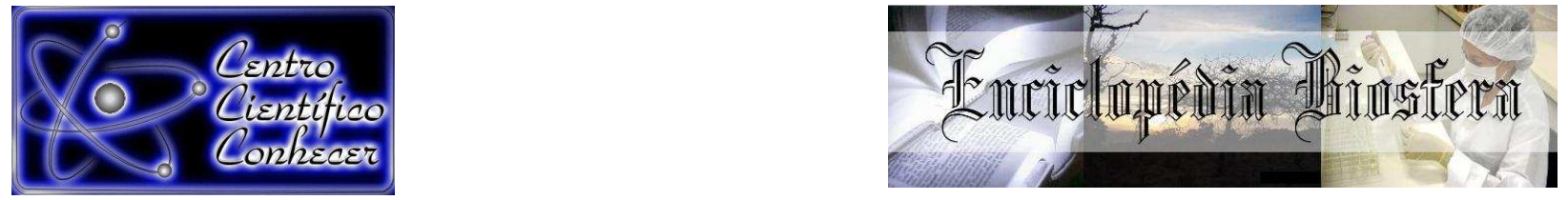

\title{
AVALIAÇÃO DE NECROMASSA EM MADEIRA MORTA EM PÉ DE UM FRAGMENTO DE FLORESTA OMBRÓFILA MISTA
}

Karina Henkel Proceke de Deus ${ }^{1}$, Izabel Passos Bonete ${ }^{1}$, Afonso Figueiredo Filho², Andrea Nogueira Dias ${ }^{2}$.

${ }^{1}$ Doutorandas em Ciências Florestais do Departamento de Engenharia Florestal da Universidade Estadual do Centro Oeste do Paraná - UNICENTRO, Paraná, Brasil karinahenkel@gmail.com

2 Professores Adjuntos do Departamento de Engenharia Florestal da Universidade Estadual do Centro Oeste do Paraná - UNICENTRO, Paraná, Brasil -

Recebido em: 03/10/2016 - Aprovado em: 21/11/2016 - Publicado em: 05/12/2016 DOI: 10.18677/EnciBio 2016B 012

As florestas desempenham um importante papel na mitigação do efeito-estufa. Durante o crescimento, as árvores captam e estocam $\circ \mathrm{CO}_{2}$ presente na atmosfera e o convertem em biomassa. A biomassa morta ou necromassa derivada da madeira morta em pé ocorre de forma natural e desempenha importante papel em ecossistemas florestais. Além de assegurar a estabilização do solo, a manutenção dos ciclos de nutrientes e o fornecimento de habitats para uma grande variedade de organismos, a necromassa florestal também contribui no combate ao efeito estufa e às mudanças climáticas. A análise desse compartimento florestal pode ser realizada pelas suas características qualitativas e quantitativas, como dimensões, espécies e grau de decomposição. Quantificar o estoque e estimar o peso do carbono estocado neste compartimento florestal tem papel fundamental para se avaliar a dinâmica florestal. Assim, o presente estudo teve como objetivo avaliar qualitativa e quantitativamente a necromassa em madeira morta em pé de um fragmento de Floresta Ombrófila Mista localizada no sul do Brasil.

PALAVRAS-CHAVE: amostragem, estoque de $\mathrm{CO}_{2}$, volume de madeira morta.

\section{NECROMASS EVALUATION IN DEAD WOOD STANDING OF A FRAGMENT OF MIXED RAIN FOREST}

\begin{abstract}
Forests play an important role in mitigating the greenhouse effect. During the growth, trees capture and store $\mathrm{CO}_{2}$ in the atmosphere and convert it into biomass. The dead biomass or necromass derived from standing dead wood occurs naturally and plays an important role in forest ecosystems. Besides ensuring soil stabilization, maintenance of nutrient cycles and providing habitats for a wide variety of organisms, forest necromass also contributes to combating global warming and climate change. The analysis of this forest module can be carried out by its qualitative and quantitative characteristics, such as size, species and degree of decomposition. Quantify the stock and estimate the carbon weight stored in this forest compartment has a key role to assess forest dynamics. Thus, this study aimed to evaluate
\end{abstract}


qualitatively and quantitatively the necromass in standing dead wood of a fragment of Araucaria Forest located in southern Brazil.

KEYWORDS: sampling, $\mathrm{CO}_{2}$ stock, dead wood volume.

\section{INTRODUÇÃO}

A biomassa morta ou necromassa compreende as árvores mortas em pé, as árvores tombadas e a serapilheira composta por galhos, folhas, flores, frutos e sementes caídos sobre o solo (MOTA \& TOREZAN, 2013). A madeira morta abrange toda a biomassa lenhosa não viva em pé, caída no chão ou no solo, com diâmetro superior a $2 \mathrm{~cm}$ (HIGA et al., 2014). Ainda que estudos sobre o compartimento florestal madeira morta sejam escassos, ALMEIDA et al. (2016) destacam que tal condição contribui para a valorização florestal, pois é fundamental para a sobrevivência de um grande número de espécies, além de ser responsável por produção de energia e armazenamento de carbono.

VEIGA (2010) reforça sobre a importância da madeira morta para os estoques de carbono e nutrientes no ciclo das florestas, destacando que após a completa decomposição da madeira, esta passa a servir de fonte de nutrientes e carbono para o ambiente. Sendo material orgânico morto, a necromassa auxilia na redução da erosão e no desenvolvimento da cobertura vegetal superficial, armazenagem de água, energia, nutrientes e substratos para 0 desenvolvimento de plântulas (ALMEIDA et al., 2013). O conhecimento dos processos de produção, deposição e acúmulo de necromassa possibilita a compreensão da dinâmica do carbono em florestas, pois ao morrer, o vegetal deixa de acumular carbono e passa a liberá-lo para o solo e para a atmosfera (MOTA \& TOREZAN, 2013).

Segundo FIORINI (2012) a diferença entre os estoques de necromassa em ecossistemas florestais pode ser um indicativo de transformações sofridas pela biomassa aérea como consequência da intensidade de distúrbios, da taxa de mortalidade natural das árvores e da taxa de decomposição da matéria orgânica na área impactada. Após um evento de distúrbio há um aumento da quantidade de necromassa, que decaí exponencialmente ao longo do tempo através do processo de decomposição.

RIBEIRO et al. (2012) destacam que a quantificação de necromassa proveniente de inventários florestais se torna cada vez mais importante, tendo em vista sua intensa ligação com o efeito estufa e com as mudanças climáticas, tornando-se um atributo essencial para um manejo florestal eficiente. A madeira morta em ecossistemas florestais pode ser avaliada por suas caraterísticas qualitativas como as dimensões, a origem (espécie ou grupo taxonômico) e o grau de decomposição (ALMEIDA et al., 2016) e por suas características quantitativas como volume, peso de necromassa e peso de carbono. Assim o presente estudo buscou avaliar, qualitativa e quantitativamente, a madeira morta em pé de um fragmento de Floresta Ombrófila Mista localizado no Sul do Brasil.

\section{MATERIAL E MÉTODOS}

A área da pesquisa está inserida na Floresta Nacional de Irati (FLONA de Irati), situada no município de Irati, região centro-sul do Paraná, com características favoráveis a realização de estudos ecológicos da fitofisionomia Floresta Ombrófila Mista (MAZZA et al., 2005).

Os dados foram coletados em parcelas permanentes instaladas em um remanescente com 1.272,90 ha, mantido sem intervenção há pelo menos 70 anos. 
As parcelas permanentes são constituídas por 25 blocos de 1 ha $(100 \mathrm{~m} \times 100 \mathrm{~m})$, que são divididas em parcelas com 0,25 ha (50 m x $50 \mathrm{~m}$ ).

O método de amostragem utilizado foi área fixa com 17 unidades amostrais de $2.500 \mathrm{~m}^{2}(50 \mathrm{~m} \times 50 \mathrm{~m})$ que totalizam uma área amostrada de 4,25 ha. Foram contabilizados apenas indivíduos mortos em pé com diâmetro à altura do peito $\geq 10$ $\mathrm{cm}$, e altura residual do fuste $\geq 1,30 \mathrm{~m}$.

As árvores mortas em pé foram identificadas conforme a espécie e família, pelas placas com numeração que possuíam resultantes do inventário das parcelas permanentes, realizado anteriormente. As árvores que não puderam ser identificadas foram agrupadas e denominadas de Não Identificadas (NI).

Conforme características apresentadas decorrentes do estágio de decomposição, as árvores mortas em pé foram classificadas de forma tátil visual, segundo características adaptadas de WOLDENDORP et al. (2002), conforme Quadro 1.

QUADRO 1 - Classes de decomposição utilizadas para árvores mortas em pé

\begin{tabular}{cl}
\hline $\begin{array}{c}\text { Classe de } \\
\text { decomposição de } \\
\text { árvore morta em pé }\end{array}$ & \multicolumn{1}{c}{ Descrição da classe } \\
\hline D1 & $\begin{array}{l}\text { Material novo - Apresenta alguns galhos, casca presente e } \\
\text { aparentemente intacta e fuste rígido. } \\
\text { Decomposição inicial - Casca solta e/ou parcialmente ausente, alguns } \\
\text { galhos presentes, grande parte coroa quebrado, fuste ainda firme. } \\
\text { Decomposição intermediária - Alguns galhos presentes ou não, topo } \\
\text { pode estar quebrado }(>2 \mathrm{~m} \text { de altura residual), casca geralmente } \\
\text { ausente e fuste ainda em pé, mas com alguns danos. } \\
\text { Decomposição avançada - Galhos ausentes, casca ausente ou pouca } \\
\text { casca, Topo quebrado }(\leq 2 \mathrm{~m} \text { de altura residual) e fortemente } \\
\text { deteriorado, fuste com cavidades extensas e ocas. }\end{array}$ \\
\hline D4 &
\end{tabular}

Fonte: adaptado de WOLDENDORP et al. (2002).

O volume de árvores mortas em pé foi obtido pela equação (1) de espécies folhosas desenvolvida no Inventário do Pinheiro do Sul do Brasil pela FUPEF (1978):

$$
v=0,0000596 \cdot d^{2,14581} h^{0,71915}
$$

Em que: $v$ = volume de árvore morta em pé $\left(\mathrm{m}^{3}\right) ; \mathrm{d}=$ diâmetro à altura do peito com casca $(\mathrm{cm}) ; \mathrm{h}=$ altura residual $(\mathrm{m})$.

Para obter o peso seco da necromassa foram adotadas densidades diferentes, conforme o grau de decomposição da madeira. A densidade utilizada foi adaptada de VIEIRA et al. (2011), sendo para D1 $\left(0,51 \mathrm{~g} \cdot \mathrm{cm}^{-3}\right)$, para D2 $\left(0,40 \mathrm{~g} . \mathrm{cm}^{-3}\right)$, para D3 $(0,36$ g. $\left.\mathrm{cm}^{-3}\right)$ e para D4 $\left(0,30 \mathrm{~g} \cdot \mathrm{cm}^{-3}\right)$. O peso da necromassa $(\mathrm{N})$ de árvores mortas em pé foi calculado, conforme RÜGINTZ et al. (2009) utilizando-se a expressão (2):

$$
\mathrm{N}=\mathrm{v} \cdot \mathrm{D}_{\mathrm{b}}
$$

Em que: $\mathrm{N}=$ Necromassa $\left(\mathrm{Mg} \cdot \mathrm{ha}{ }^{-1}\right)$ de árvores mortas em pé; $\mathrm{v}=$ Volume $\left(\mathrm{m}^{3} \cdot \mathrm{ha}^{-1}\right)$ de árvores mortas em pé; $D_{b}=$ Densidade básica da madeira morta $\left(\mathrm{g} \cdot \mathrm{cm}^{-3}\right)$.

Para obter o peso de carbono $(\Delta \mathrm{C})$, aplicou-se a expressão (3):

$$
\Delta \mathrm{C}=\mathrm{N} . \mathrm{CF}
$$


Em que: $\Delta \mathrm{C}=$ quantidade de carbono na necromassa $\left(\mathrm{Mg} \mathrm{C}\right.$.ha $\left.{ }^{-1}\right) ; \mathrm{N}=$ necromassa de árvores mortas em pé (Mg.ha); $\mathrm{CF}=$ fração de carbono $=0,475$ (conforme recomendação de MAGNUSSEN \& REED (2004).

\section{RESULTADOS E DISCUSSÃO}

Foram encontradas 101 árvores mortas em pé com DAP $\geq 10 \mathrm{~cm}$, o que equivale a 23,76 árvores/hectare. Segundo ROCHA (2011) é normal ser encontrado, no interior das florestas brasileiras, um grande número de árvores mortas resultantes de morte natural, acidente, parasitismo ou perturbações antrópicas. RIBEIRO et al. (2012) em uma área de 9,5 ha de um remanescente de Floresta Ombrófila Mista, encontraram 437 árvores/hectare, um valor relativamente alto, porém foram consideradas todas as árvores em pé com diâmetro acima de três centímetros. GASPER et al. (2013) avaliaram 143 unidades amostrais em um fragmento de Floresta Ombrófila Mista localizado em Santa Catarina e encontraram 1.860 árvores mortas, o que equivale a uma taxa média de mortalidade de 5,8\% e média de 60 árvores/hectare.

Com relação às características das árvores mortas em pé, foram registradas 30 espécies. As espécies encontradas foram descritas por nome científico, nome vulgar, família, número de indivíduos por classe de decomposição e número total de árvores/hectare (Tabela 1).

TABELA 1 - Número de árvores mortas em pé por hectare (N/ha), separadas por espécie, família e classe de decomposição.

\begin{tabular}{|c|c|c|c|c|c|c|c|}
\hline \multicolumn{3}{|c|}{ Espécie } & \multicolumn{4}{|c|}{$\begin{array}{c}\text { Classes de } \\
\text { decomposição }\end{array}$} & \multirow{2}{*}{$\begin{array}{l}\text { Total } \\
\text { (N/ha) }\end{array}$} \\
\hline Nome científico & Nome vulgar & Família & D1 & D2 & D3 & D4 & \\
\hline A. edulis & Vacum & Sapindaceae & - & 0,24 & - & - & 0,24 \\
\hline C. decandra & Guaçatunga-branca & Salicaceae & 0,24 & 0,24 & - & - & 0,48 \\
\hline C. dinisii & Pimenteira & Canellaceae & - & 0,24 & - & - & 0,24 \\
\hline C. fissilis & Cedro & Meliaceae & - & 0,24 & - & - & 0,24 \\
\hline C. obliqua & Guaçatunga-vermelha & Salicaceae & - & - & 0,24 & - & 0,24 \\
\hline C. scabra & Carne-de-vaca & Clethraceae & 0,47 & - & - & - & 0,47 \\
\hline C. sellowianum & Canela-branca & Lauraceae & - & 0,24 & - & - & 0,24 \\
\hline C. sylvestris & Guaçatunga-preta & Salicaceae & - & - & 0,24 & - & 0,24 \\
\hline C. xanthocarpa & Guabiroba & Myrtaceae & - & 0,47 & - & - & 0,47 \\
\hline C.contracta & Cinzeiro-preto & Rubiaceae & - & - & 0,24 & - & 0,24 \\
\hline D. brasiliensis & Jacarandá & Fabaceae & 0,24 & 0,24 & - & 0,24 & 0,72 \\
\hline I. dumosa & Cauninha & Aquifoliaceae & - & - & 0,24 & - & 0,24 \\
\hline I. paraguariensis & Erva-mate & Aquifoliaceae & - & 0,24 & 0,24 & - & 0,48 \\
\hline I. theezans & Caúna & Aquifoliaceae & 0,24 & - & 0,47 & - & 0,71 \\
\hline L. fruticosa & Santa-Rita & Theaceae & - & - & 0,24 & - & 0,24 \\
\hline M. elaeagnoides & Miguel-pintado & Sapindaceae & - & 0,47 & 0,24 & - & 0,71 \\
\hline M. hebepetala & Caingá & Myrtaceae & - & - & 0,24 & - & 0,24 \\
\hline M. splendens & Guamirim-preto & Myrtaceae & 0,24 & 0,47 & 0,71 & - & 1,42 \\
\hline M. umbellata & Capororocão & Primulaceae (Myrsinaceae) & 0,47 & 0,47 & 1,18 & - & 2,12 \\
\hline N. grandiflora & Canela-amarela & Lauraceae & - & 0,24 & 0,47 & - & 0,71 \\
\hline N. megapotamica & Canela-imbuia & Lauraceae & 0,24 & - & - & - & 0,24 \\
\hline$N I$ & $\mathrm{NI}$ & - & - & 0,24 & 6,59 & 2,82 & 9,65 \\
\hline O. odorifera & Sassafrás & Lauraceae & - & - & 0,71 & - & 0,71 \\
\hline O. puberula & Canela-guaicá & Lauraceae & - & - & 0,24 & - & 0,24 \\
\hline$P$. angustifolia & Vassourão-branco & Asteraceae & - & - & 0,24 & - & 0,24 \\
\hline P. crucis & Cambroé & Salicaceae & 0,24 & - & 0,24 & - & 0,48 \\
\hline P. myrtifolia & Pessegueiro-bravo & Rosaceae & 0,24 & - & 0,24 & - & 0,48 \\
\hline$P$. vellosiana & Jasmim-verdadeiro & Rubiaceae & - & - & 0,24 & - & 0,24 \\
\hline S. hirsuta & Sapopema & Elaeocarpaceae & 0,24 & - & - & - & 0,24 \\
\hline S. romanzoffiana & Palmeira & Arecaceae & - & - & 0,47 & - & 0,47 \\
\hline V. petiolaris & Vassourão & Asteraceae & - & - & 0,24 & - & 0,24 \\
\hline & Total & & 2,86 & 3,80 & 13,9 & 3,06 & 23,76 \\
\hline
\end{tabular}

$\mathrm{NI}$ : Não identificadas 
Constatou-se que a maior frequência de árvores mortas em pé correspondeu ao grupo de árvores não identificadas (9,65 árvores/hectare), equivalente a 40,6\% do total. Das árvores não identificadas, $68,3 \%$ estavam em estágio de decomposição intermediária (D3) com alguns galhos presentes, topos quebrados, alturas residuais maiores que $2 \mathrm{~m}$, cascas geralmente ausentes e fustes ainda em pé, mas com alguns danos.

O Capororocão, embora em menor proporção (2,12 árvores/hectare), apresentou-se como uma das espécies identificadas mais frequentes, representando $8,92 \%$ do total e com $55,66 \%$ em estágio de decomposição intermediária. Quanto à decomposição da madeira morta, VEIGA (2010) destaca que o processo depende da espécie, da densidade da madeira, do tamanho da madeira morta, da área de contato com o solo, das condições edáficas como fertilidade do solo e presença de minerais, mas principalmente, pelas condições climáticas.

As espécies menos frequentes, com 0,24 árvores/ha, mas em diferentes estágios de decomposição foram: Vacum, Pimenteira, Cedro, Guaçatunga-vermelha, Canela-branca, Guaçatunga-preta, Cinzeiro-preto, Cauninha, Santa Rita, Caingá, Canela imbuia, Canela-guaicá, Vassourão-branco, Jasmim-verdadeiro, Sapopema e Vassourão.

O volume estimado correspondeu a $11,12 \mathrm{~m}^{3} \cdot \mathrm{ha}^{-1}$ (Tabela 2), sendo o maior estoque $\left(5,53 \mathrm{~m}^{3} \cdot \mathrm{ha}^{-1}\right)$, correspondente a madeira morta em pé em estágio de decomposição intermediária (D3) e o menor volume $\left(0,59 \mathrm{~m}^{3}\right.$.ha $\left.{ }^{-1}\right)$ correspondente a árvores mortas em pé em estágio de decomposição avançada (D4).

TABELA 2 - Volume e percentual de árvore morta em pé por classe de decomposição na modalidade árvore morta em pé.

\begin{tabular}{ccc}
\hline Classe de Decomposição & Volume $\left(\mathbf{m}^{\mathbf{3}} \cdot \mathbf{h a}^{-1}\right)$ & $\%$ \\
\hline D1 & 2,75 & 24,72 \\
D2 & 2,24 & 20,17 \\
D3 & 5,53 & 49,78 \\
D4 & 0,59 & 5,33 \\
\hline Total & $\mathbf{1 1 , 1 2}$ & $\mathbf{1 0 0 , 0 0}$ \\
\hline
\end{tabular}

D1: Classe de decomposição de material novo; D2: Classe de decomposição inicial; D3: Classe de decomposição intermediária; D4: Classe de decomposição avançada.

Ao realizar inventário de madeira morta em remanescente de floresta ombrófila mista na cidade de Curitiba, RIBEIRO et al. (2012) obtiveram um volume de $10,99 \mathrm{~m}^{3}$.ha ${ }^{-1}$ para árvores mortas em pé com diâmetro acima de $3 \mathrm{~cm}$. Nas regiões de Santa Catarina, CARDOSO et al. (2012) estimaram um volume de 5,6 $\mathrm{m}^{3}$.ha ${ }^{-1}$ para árvores mortas com diâmetro mínimo de $1 \mathrm{~cm}$. Ambos os resultados foram inferiores ao estimado neste trabalho que considerou um limite maior de inclusão do diâmetro.

Entretanto, um volume médio de $28,28 \mathrm{~m}^{3}$.ha ${ }^{-1}$ foi encontrado por NEVES \& PINTO (2011) em floresta primária do estado do Amazonas em uma área de 6 ha, em que foram medidos todos os indivíduos mortos em pé com DAP $\geq 10 \mathrm{~cm}$.

O maior peso de necromassa e de carbono estocado, estimados no presente estudo, foram de árvores mortas em pé em decomposição intermediária (D3), a classe que apresentou maior volume e percentual de árvore morta (Tabela 3). MOTA \& TOREZAN (2013) também estimaram a biomassa dos troncos por meio da multiplicação do volume pela densidade da madeira e concluíram que quanto maior a deposição de necromassa, maior a concentração de carbono orgânico no solo. 
TABELA 3 - Peso da Necromassa e peso de carbono de árvores mortas em pé por classe de decomposição

\begin{tabular}{c|c|c}
\hline Classe de decomposição & $\begin{array}{c}\text { Peso de necromassa } \\
\text { (Mg.ha' }^{-1} \text { ) }\end{array}$ & $\begin{array}{c}\text { Peso de carbono } \\
\text { (Mg.C.ha }^{-1} \text { ) }\end{array}$ \\
\hline D1 & 1,40 & 0,67 \\
D2 & 0,90 & 0,43 \\
D3 & 1,99 & 0,95 \\
D4 & 0,18 & 0,08 \\
\hline Total & $\mathbf{4 , 4 7}$ & $\mathbf{2 , 1 2}$ \\
\hline
\end{tabular}

D1: Classe de decomposição de material novo; D2: Classe de decomposição inicial; D3: Classe de decomposição intermediária; D4: Classe de decomposição avançada.

FIORINI (2012) ao avaliar estoque de necromassa ao longo de um gradiente de degradação florestal no município de Santarém na Amazônia Brasileira, obteve 37,7 Mg.ha' ${ }^{-1}$, distribuídos em áreas de florestas não perturbadas (9,2 Mg.ha $\left.{ }^{-1}\right)$, florestas com marcas de extração $\left(8,8 \mathrm{Mg} \mathrm{ha}^{-1}\right)$, florestas com marcas de extração e fogo (7,9 Mg.ha $\left.{ }^{-1}\right)$, florestas queimadas(8,4 Mg.ha $\left.{ }^{-1}\right)$ e de florestas secundárias $(3,4$ Mg.ha' $\left.{ }^{-1}\right)$.

Com relação ao peso de carbono, SANQUETA et al. (2014) obtiveram 4,44 Mg.ha ${ }^{-1}$ de estoque médio em necromassa lenhosa proveniente de povoamentos de Pinus taeda no centro-sul do Estado do Paraná e VIEIRA et al. (2011) estimaram um peso de carbono de 2,28 Mg.C.ha ${ }^{-1}$ em floresta atlântica montana na costa brasileira, resultados semelhantes ao encontrado no presente estudo (Tabela 3).

\section{CONCLUSÕES}

Constatou-se que apenas 2,86 árvores mortas em pé/hectare se encontravam em estágio de decomposição inicial (D1) e que a maior parte delas (13,9 árvores/ha) se encontravam em estágio de decomposição intermediária (D3).

Entretanto, considerando o volume de necromassa encontrado $(11,12$ $\mathrm{m}^{3}$.ha $\mathrm{h}^{-1}$ ), bem como o peso de necromassa $\left(4,47 \mathrm{Mg} \mathrm{ha}^{-1}\right)$ e o peso de carbono (2,12 Mg.C.ha $\left.{ }^{-1}\right)$, é possível concluir que as árvores mortas em pé contribuem, mesmo que em menor proporção que a biomassa viva, para a redução da concentração do $\mathrm{CO}_{2}$ na atmosfera e, consequentemente, para a diminuição do efeito estufa, um dos maiores desafios da humanidade.

\section{REFERÊNCIAS}

ALMEIDA, A. R.; NOVAIS, A. T.; FLÁVIO, H.; OLIVEIRA, S. da S.; PEREIRA, R.; SANTOS, P. P. T. Madeira morta: uma revisão da importância da sua conservação. Captar, v. 6, n.2. p. 32-43, Porto, Portugal, 2016. Disponível em < http://revistas.ua.pt/index.php/captar/article/view/3655/3369>

ALMEIDA, E. J.; VALÉRIO, B. E.; NORONHA, J. C.; MIRANDA, R. M.; BARBOSA, L. F.; MACHINER, M.; PEZZINI, L. A.; CABECEIRA, F. G.; LIMA, D. C.; RODRIGUES, D. J. Estimativa de necromassa em área de floresta na Amazônia Meridional a partir de duas metodologias distintas. Anais do II Simpósio Cenbam e PPBIO Amazônia Ocidental. Manaus, 2013. Disponível em: < https://ppbio.inpa.gov.br/sites/default/files/BANNERS_ABIOTICOS.pdf>

CARDOSO, D. J.; VIBRANS, A. C.; LINGNER, D. V. Inventário da necromassa florestal caída no chão nos remanescentes florestais de Santa Catarina. In: VIBRANS, A. C.; SEVEGNANI, L.; GASPER, A. L. de; LINGNER, D. V. (Ed.). 
Inventário Florístico Florestal de Santa Catarina. Blumenau: FURB, p.217- 227, 2012.

em:

https://www.researchgate.net/profile/Andre_De_Gasper/publication/235632008_Volu me_I_-

Inventario_Floristico_Florestal_de_Santa_Catarina/links/00b49515f5256702460000 00.pdf?origin=publication_detail>

FIORINI, A.C.O. A importância da madeira morta para estimar estoques de carbono em florestas degradadas: implicações para ações de redução de emissão por desmatamento e degradação florestal. (137f.). Dissertação (mestrado)-UFRJ/COPPE/Programa de Planejamento Energético, 2012. Disponível em: < www.redeamazoniasustentavel.org/app/download/.../Fiorini+2011+MSc.pdf>

FUNDAÇÃO DE PESQUISAS FLORESTAIS DO PARANÁ (FUPEF). 1978. Inventário florestal do Pinheiro no sul do Brasil. Curitiba, 327 p.

GASPER, A. L.; MEYER, L.; LINGNER, D.V.; VIBRANS, A. C.; SEVEGNANI, L.; UHLMANN, A. Quantidade de árvores mortas em pé nas florestas de Santa Catarina. Anais 64ํㅡㄹ Congresso Nacional de Botânica Belo Horizonte, 10-15 de Novembro de 2013. Disponível em: < https://www.alice.cnptia.embrapa.br/alice/bitstream/doc/976642/1/2013AlexandreCN BotQuantidade.pdf>

HIGA, R. C. V.; CARDOSO, D. J.; ANDRADE, G.; ZANATTA, J. A.; ROSSI, L. M. B.; PULROLNIK, K.; NICODEMO, M. L.; GARRASTAZU, M. C.; VASCONCELOS, S.; SALIS, S. M. de. Protocolo de medição e estimativa de biomassa e carbono florestal. Colombo: Embrapa Florestas, 2014. 68 p. (Embrapa Florestas. Documentos, 266).

MAGNUSSEN, S.; REED, D. Modelling for estimation and monitoring. Knowledge Reference for National Forest Assessments: FAO-IUFRO, 2004. Disponível em: < http://www.fao.org/fileadmin/user_upload/national_forest_assessment/images/PDFs/ English/KR2_EN__10_.pdf>

MAZZA, C. A. da S.; MAZZA, M. C. M.; SANTOS, J. E. SIG Aplicado à Caracterização Ambiental de uma Unidade de Conservação Floresta Nacional de Irati, Paraná. In: Anais XII Simpósio Brasileiro de Sensoriamento Remoto, Goiânia, Brasil, 16-21, INPE, p. 2251-2258, abril 2005. Disponível em: < http://marte.sid.inpe.br/col/ltid.inpe.br/sbsr/2004/10.26.14.06/doc/2251.pdf>

MOTA, M. C.; TOREZAN, J. M. D. Necromassa em reflorestamentos com espécies nativas da Mata Atlântica com 4, 6 e 8 anos de implantação. Hoehnea 40(3): 499505, 2013. Disponível em: < http://www.scielo.br/pdf/hoehnea/v40n3/09.pdf>

NEVES, P. R. G.; PINTO, F. R. Estoque de carbono da necromassa em pé no Rio Arari, Itacoatiara- AM. Anais do X Congresso de Ecologia do Brasil, 16 a 22 de Setembro de 2011, São Lourenço - MG. Disponível em: <http://www.sebecologia.org.br/xceb/resumos/2168.pdf> 
RIBEIRO, A.; PÉLliCO NETTO, S.; STALL, D.; LEÃO, R. A.; NASCIMENTO, F. A. F. do. Proposta metodológica para realização de um inventário florestal de necromassa: um estudo de caso. Scientia Forestalis, Piracicaba, v. 40, n. 93, p. 121-127, mar. 2012. Disponível em: < http://www.bibliotecaflorestal.ufv.br:80/handle/123456789/15885>

ROCHA, C. C. C. Estimativa de volume, biomassa e carbono da vegetação lenhosa de floresta estacional no estado de Goiás. (80 f.). Dissertação (mestrado). Programa de Pós-graduação em Ciências Florestais. Universidade de Brasília, 2011. Disponível em: < http://repositorio.unb.br/handle/10482/9345>

SANQUETA, C. R.; CORTE, A. P. D.; BARBEIRO, L. S. S.; MAAS, G. C. B.; SANQUETTA, M. N. I. Estoques de necromassa e carbono em povoamentos de Pinus taeda L. no centro-sul do estado do Paraná. Enciclopédia Biosfera, Centro Científico Conhecer - Goiânia, v.10, n.18; p. 2014. Disponível em: < http://www.conhecer.org.br/enciclop/2014a/AGRARIAS/Estoques.pdf>

RÜGNITZ, M. T.; CHACÓN, M. L.; PORRO R. Guia para Determinação de Carbono em Pequenas Propriedades Rurais. 1a ed. Belém, Brasil. Centro Mundial Agroflo-restal (ICRAF)/ Consórcio Iniciativa Amazônica (IA). P.81. 2009. Disponível em: < http://www.worldagroforestry.org/sites/default/files/GuiaCarbonPortug.pdf>

VEIGA, L. G.. Estoque de madeira morta ao longo de um gradiente altitudinal de Mata Atlântica no nordeste do estado de São Paulo. (82f.). Dissertação (Mestrado). Universidade Estadual de Campinas-SP, 2010. Disponível em: < http://www2.ib.unicamp.br/profs/cjoly/0\%20-

$\% 20$ Produ\%E7\%E30\%20Tematico/2\%20-

\%20Disserta\%E7\%F5es/2010/VEIGA,\%20L.G\%202010\%20UNICAMP.pdf>

VIEIRA, S. A; ALVES, L. F.; DUARTE-NETO, P. J.; MARTINS, S. C.; VEIGA, L. G.; SCARANELLO, M. A.; PICOLLO, M. C.; CAMARGO, P. B.; CARMO, J. B. DO; NETO, E. S.; SANTOS, F. A. M., JOLY, C. A.; MARTINELLI, L. A. Stocks of carbon and nitrogen and partitioning between above- and belowground pools in the Brazilian coastal Atlantic Forest elevation range. Ecology and evolution, v. 1, n. 3, p. 42134, 2011. Disponível em: < https://www.ncbi.nlm.nih.gov/pmc/articles/PMC3287305/> Doi: $10.1002 /$ ece3.41

WOLDENDORP, G.; SPENCER, R. D.; KEENAN, R. J. An Analysis of Sampling Methods for Coarse Woody Debris in Australian Forest Ecosystems. v.6, 84 p., nov. $2002 . \quad$ Disponível em: < http://citeseerx.ist.psu.edu/viewdoc/download?doi=10.1.1.161.170\&rep=rep1\&type=p $\mathrm{df}>$ 